\title{
Tim Schanetzky, «Kanonen statt Butter ». Wirtschaft und Konsum im Dritten Reich
}

\section{Charlotte Pouly}

\section{OpenEdition}

Journals

Édition électronique

URL : http://journals.openedition.org/ifha/8652

DOI : $10.4000 /$ ifha. 8652

ISSN : 2198-8943

Éditeur

IFRA - Institut franco-allemand (sciences historiques et sociales)

Référence électronique

Charlotte Pouly, «Tim Schanetzky, "Kanonen statt Butter ». Wirtschaft und Konsum im Dritten Reich», Revue de l'IFHA [En ligne], Date de recension, mis en ligne le 01 février 2017, consulté le 24 septembre 2020. URL : http://journals.openedition.org/ifha/8652 ; DOI : https://doi.org/10.4000/ifha.8652

Ce document a été généré automatiquement le 24 septembre 2020

(CIFHA 


\section{Tim Schanetzky, « Kanonen statt Butter ». Wirtschaft und Konsum im Dritten Reich}

Charlotte Pouly

\section{RÉFÉRENCE}

Tim Schanetzky, « Kanonen statt Butter ». Wirtschaft und Konsum im Dritten Reich, München: Verlag C.H Beck, 2015, 272 p., 16,95€ 
«Et nous sommes prêts aussi à l'avenir, si nécessaire, à consommer encore un peu moins de graisse, un peu moins de viande de porc et d'œufs [...]. Nous savons que les devises, que nous allons ainsi économiser, se révèleront être un avantage pour l'armement. Aussi le slogan en vigueur aujourd'hui est : des canons plutôt que du beurre ». Ainsi énoncé par Rudolf Hess au mois d'octobre 1936, le slogan nazi "Kanonen statt Butter» va rapidement se propager à la radio, dans la presse, lors des congrès politiques et des fêtes sportives du NSDAP pour devenir l'un des symboles de la politique économique et militaire du III $^{\mathrm{e}}$ Reich. Tim Schanetzky, dont ce n'est pas là le premier ouvrage sur l'économie national-socialiste (voir Unternehmen im Nationalsozialismus. Zur Historisierung einer Forschungskonjunktur,

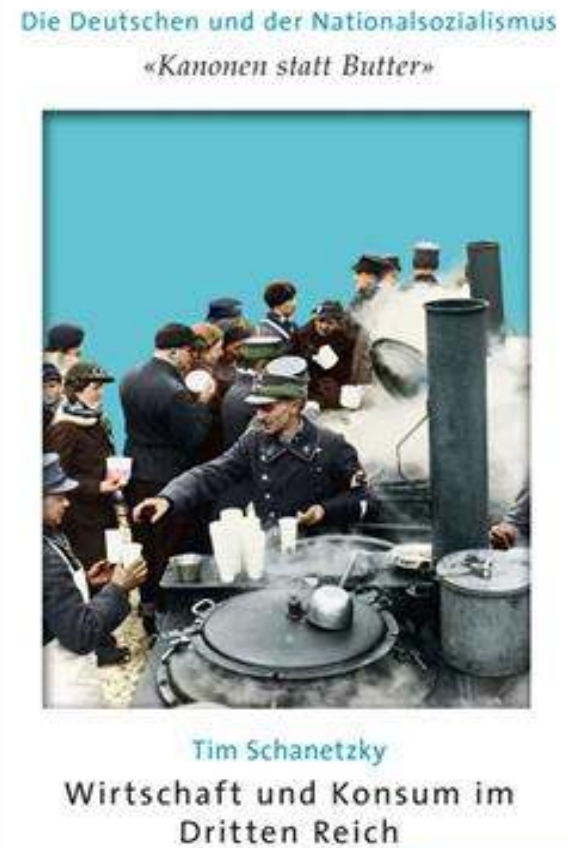

C.H.Beck 2010), en fait le fil conducteur de ce livre qui propose une « histoire moderne de la société allemande sous le national-socialisme", conformément à l'objectif de la collection dans lequel il s'inscrit («Die Deutschen und der Nationalsozialismus », dirigée par Norbert Frei depuis 2015 chez C.H Beck).

La société allemande à l'époque du NSDAP y est abordée à travers la problématique, encore relativement peu traitée, de la consommation civile allemande ( Butter») dans un contexte de crise économique où le nouveau régime dictatorial fait le choix du réarmement et de la guerre ("Kanonen »). Le livre se situe donc à la croisée de deux historiographies : celle, déjà très dense, de l'économie et des entreprises pendant la Seconde Guerre mondiale (on peut lire en français le numéro 4/volume 25 de la revue Histoire, Economie et Société consacré aux «Entreprises allemandes durant la Seconde Guerre mondiale » paru en 2005 ou encore l'excellent ouvrage d'Adam Tooze, Le Salaire de la destruction. Formation et ruine de l'économie nazie, 2012) et celle, encore en construction car plus récente, de l'histoire de la consommation allemande (voir en français Heinz-Gerhard Haupt, « Pour une histoire de la consommation en Allemagne » dans Le Mouvement social, $\mathrm{n}^{\circ} 206,1 / 2004$, p. 3-16).

Le lecteur trouvera sous la plume de T. Schanetzky non pas un ouvrage de pur style académique reposant sur des recherches empiriques denses et variées destinées à étayer une nouvelle thèse, mais une synthèse historique s'adressant à un large public, conformément à l'objectif de la collection dans laquelle s'insère ce second opus : les sources primaires cèdent la place à une littérature abondante, le contenu est aisément accessible aux non spécialistes et ce petit ouvrage, à la limite de l'essai, épargne aux néophytes les questions techniques (la construction des statistiques de la production, de l'approvisionnement et de la consommation des ménages, par exemple) et les controverses historiographiques (sur les marges de manœuvre des entreprises, sur la question du primat entre la sphère politique et le champ économique, mais aussi sur les 
ruptures et les continuités dans l'histoire de la consommation allemande des années 1920-1930 aux années 1950-1960). Composée d'une introduction, d'une conclusion, d'un petit index et de cinq chapitres, cette synthèse suit un raisonnement chronothématique : les trois premiers chapitres sont consacrés à la période 1933-1939 et seuls les deux derniers abordent la période de guerre stricto sensu. En guise d'introduction, T. Schanetzky retrace le cheminement et l'histoire du slogan « Kanonen statt Butter » qui illustre parfaitement la crise d'approvisionnement touchant les Allemands à partir de 1933 et les réponses apportées par le nouveau régime marqué du sceau du Führerprinzip. Dès 1935-1936, les ténors du régime national-socialiste, à commencer par Joseph Goebbels chargé de la propagande, suivi du responsable du Plan de 4 ans Hermann Göring, s'emparent du problème de la crise économique, du chômage, de l'inflation, de la pénurie et du rationnement de certaines denrées telles que le beurre, la viande et le pain pour servir non seulement des objectifs économiques et militaires à visée expansionniste et autarcique, mais aussi et surtout les intérêts politiques, idéologiques et racistes du NSDAP. Pour une nation en recomposition (d'après l'idée de la Volksgemeinschaft qui représente, selon T. Schanetzky, davantage qu'une simple propagande de forme) que l'on souhaite préparer au réarmement, à la guerre et à l'autarcie dans un contexte de crise économique mondiale et de concurrence entre grandes puissances, il s'agit d'accorder le primat au secteur militaire sans pour autant négliger l'opinion publique puisque la société allemande subit quotidiennement, et de plein fouet, les restrictions imposées. Ici, le calcul économique est si étroitement lié aux stratégies militaire, politique et idéologique, pour un parti construit sur une base populiste et élu avec la promesse d'une amélioration du niveau de vie des ménages, que la propagande dépasse aisément le stade discursif pour acquérir très vite une dimension performative. À peine avait-il été énoncé que le slogan Kanonen statt Butter faisait déjà partie du langage nazi.

Dans un premier chapitre consacré à l'année 1933, "Terreur et promesse », l'auteur aborde l'aryanisation précoce et le boycott des commerces juifs qu'il qualifie de «faillite morale». S'agissant des entreprises, il nous rappelle le rôle majeur des principaux Konzerne allemands dans le financement du NSDAP, l'émergence rapide d'un modus vivendi entre le secteur privé et les politiques économiques publiques nazies et, enfin, l'opportunisme caractéristique de certaines branches, à commencer par la chimie avec IG Farben. Ce chapitre se conclut sur la priorité accordée au travail avec la création du Deutsche Arbeitsfront (DAF, «Front allemand du travail ») et sur l'émergence de l'idéologie naturiste du retour à la terre, à l'image du slogan "Blut und Boden" (le sang et le sol) de l'agronome Richard Walther Darré, nommé ministre de l'Agriculture et de l'Alimentation en 1933.

Intitulé «Le chemin de la nécessité », le second chapitre s'étend de 1933 à la fin des années 1930. T. Schanetzky y traite des standards de vie et du pouvoir d'achat des ménages, du réarmement et du rêve d'un grand espace économique autarcique. Alors que l'investissement et la production augmentent fortement dans les secteurs clés de l'armement dès 1935, que la crise des devises et l'utopie d'une économie continentale autosuffisante aboutissent à la réduction des importations, à la bilatéralisation des échanges avec les pays de l'Est et du Sud de l'Europe (espace de la Mitteleuropa) et à l'émergence de produits de synthèse, les Allemands vivent globalement moins bien que leurs homologues français, anglais et américains. En effet, dans un contexte de chômage de masse (6 millions de chômeurs officiels en 1933), en partie régulé par de grands travaux publics (les Reichsautobahnen par exemple), $62 \%$ des contribuables 
allemands ne gagnent pas plus de 60 pfennige/heure (30 marks/semaine) en 1936. Il faut donc l'équivalent d'une demi-heure de travail pour pouvoir acheter une miche de pain, 1 heure pour acquérir une demi-livre de beurre, une demi-douzaine d'œufs ou un litre de bière et enfin le salaire d'une demi-journée de labeur pour pouvoir manger $500 \mathrm{~g}$ de lard. Si la consommation de certaines denrées alimentaires de base diminue bien, la société allemande n'est toutefois pas exclue de la consommation moderne de masse, comme en témoignent la production et l'achat d'automobiles et de postes de radio.

Dans un troisième chapitre, "Les bonnes années ", le dernier qui se focalise sur la période d'avant-guerre et peut-être le plus intéressant d'entre tous, l'auteur nous plonge dans l'univers des "mondes de consommation » et du travail, en prenant soin de différencier le monde urbain des ouvriers du monde rural et agricole. La consommation de masse sous le régime national-socialiste comporte deux caractéristiques majeures: le développement d'une culture des loisirs teintée de paternalisme, à l'image des vacances organisées par le Kraft durch Freude (« La force par la joie ») grâce aux Sonderzüge (transports de masse à tarif réduit) de la Reichsbahn, et l'essor des «Volksprodukte» qui s'insère parfaitement dans l'idéologie de la Volksgemeinschaft. Il s'agit alors de favoriser une production nationale allemande à bas coût pour équiper les foyers en Volksfernseher (poste de télévision), Volkskühlschrank (réfrigérateur) et autres produits domestiques modernes, telle la mythique « voiture du peuple » Volkswagen. Du mois d'août 1938 à la fin de la guerre, 340000 Allemands en seront équipés pour une somme inférieure à 1000 marks (l'Opel de Ford vaut alors 1500 marks) grâce à une formule d'achat parrainée par le DAF. Or, pour T. Schanetzky, le "Volksprodukt" renvoie surtout à la mise en scène d'une "pseudo société de consommation de masse » orchestrée par la propagande nazie. En effet, le régime se heurte à des investissements souvent très onéreux, le coût des articles freine l'équipement des ménages les plus modestes, certains produits peinent à se massifier (les logements ouvriers Volkswohnungen, par exemple) alors que d'autres sont portés par le régime et sa propagande et par l'industrie, de leur conception à leur mise en circulation. En outre, le «monde» et le mode de consommation ne sont guère comparables entre l'environnement urbain et la vie à la campagne, même si ouvriers et agriculteurs subissent tout autant les pressions du régime. D'après l'idéologie du «Blut und Boden " de Walther Darré, la paysannerie, pierre angulaire de la Volksgemeinschaft, représente la source de la vie face à la décadence des races induite par le mode de vie urbain. Après la crise agricole de 1932 due à la chute des prix de 1927-1928, Darré souhaite remodeler le secteur en suivant une formule coopérative afin d'atteindre l'autosubsistance. L'agriculture devient intensive, mais peine à se motoriser rapidement. Aussi, même si la production locale augmente de 1933 à 1938 parallèlement à la réduction des importations, la pénurie d'aliments gras (crème, lait, huile, beurre etc.) impose le recours à de nombreux produits de substitution (notamment la margarine à la place du beurre).

Dans les deux derniers chapitres de son livre, sans doute les moins marquants dans la mesure où les faits relatés sont déjà bien connus et largement étudiés, l'auteur analyse la période de guerre en présentant successivement les objectifs du Plan de 4 ans, la politique d'aryanisation économique et les plans nazis d'après-guerre tels que le Generalplan Ost et l'europäische Großwirtschaftsraum. Le dernier chapitre porte sur ce que T. Schanetzky nomme "l'économie de grand butin» qui se caractérise par l'exploitation systématique des ressources économiques et humaines des pays occupés, 
par l'essor d'une économie parallèle (marché noir) et par le recours massif au travail forcé. De quoi relativiser, une fois de plus et s'il en était encore besoin, le " miracle de l'approvisionnement et du réarmement » et la politique économique d'Albert Speer.

En somme, à l'échelle du Reich de 1933 à 1945, au niveau politique, idéologique et structurel, il existe bien une spécificité dans la consommation allemande orientée par le NSDAP, marquée par l'expérience du manque, la préparation à la guerre et l'idéologie du Volksprodukt. Cependant, à une échelle plus globale et sur la moyenne durée, le particularisme est à nuancer puisque ce modèle moderne de consommation de masse n'est au fond qu'une variante nationale du modèle fordiste américain, et le succès de la Volkswagen ne peut se comprendre en dehors du contexte de guerre économique entre les États-Unis et le III ${ }^{e}$ Reich continental. Si l'occupation alliée en RFA fait entrer la société allemande de l'Ouest dans l'ère de la consommation de masse dès la fin de la guerre, où l'heure est désormais aux Lucky Strike et au Coca Cola, la société allemande de l'Est devra, quant à elle, attendre encore un peu.

INDEX

Index chronologique : Époque contemporaine

Thèmes : Histoire économique, Histoire sociale, Histoire des États et des pouvoirs

\section{AUTEURS}

\section{CHARLOTTE POULY}

Institut franco-allemand/Sciences historiques et sociales (IFRA/SHS), Goethe Universität

(Frankfurt am Main) 